\title{
MUST and liver disease patients: adaptation of a validated screening tool to aid identification of malnourished individuals
}

\author{
S. C. Cooper ${ }^{1}$, J. Johnson ${ }^{2}$ and J. M. Neuberger ${ }^{2}$ \\ ${ }^{1}$ Russells Hall Hospital, Dudley Group of Hospitals NHS Foundation Trust Dudley DY1 $2 H Q$ and ${ }^{2}$ Queen Elizabeth \\ Hospital, University Hospital Birmingham NHS Foundation Trust, Edgbaston, Birmingham B15 2TH
}

Malnutrition is common among acute hospital admissions, affecting up to $40 \%$ of patients, with many deteriorating (78\%). MUST is NICE endorsed to aid identification of those at nutritional risk, validated against patient outcome in order to prevent the complications of malnutrition, and consequently reduce mortality, morbidity and cost to the health services. Liver disease patients are at particular risk of malnutrition. Lean weight loss can be masked by ascites or oedema, thus not revealing risk by use of MUST which relies, in part, upon change in weight.

We recruited patients undergoing liver transplant assessment at the Queen Elizabeth Hospital Liver Unit, University Hospital Birmingham. MUST was calculated, and anthropometrics and DEXA scans were performed. An adjustment was made to MUST, by addition of one point for each of the following: fluid overload (significant ascites or oedema), chronic clinically evident encephalopathy, clinical jaundice and diarrhoea/steatorrhoea, creating the Malnutrition Liver Universal Screening Tool score (MLUST). Spearman's test was used to identify the degree of correlation between MUST and MLUST with anthropometrics and fat mass by DEXA scan. The degree of agreement between MUST and MLUST, with identification of malnutrition by mid-arm muscle circumference (MAMC) was calculated with the kappa statistic.

Eighty nine patients with liver disease (52 male, median age 52.5, interquartile range 45.25-58) were examined. MUST and MLUST scores showed no correlation with tricep skin-fold thickness and hand-grip strength, and only MUST showed correlation with mid-arm circumference $(r=-0.26, P=0.038)$. However, both MUST and MLUST showed correlations with identification of being malnourished (including degree of malnourishment) by MAMC $r=0.25, P=0.024$ and $r=0.25, P=0.022$, respectively. MUST score showed a significant correlation with thigh region fat mass at DEXA $(r=-0.33, P=0.014)$, but not with arms or whole body; MLUST showed no correlation with DEXA. When MUST and MLUST were dichotomised into triggering referral or not, and agreement with identification of being malnourished by MAMC, MUST derived a kappa statistic of 0.16 (slight agreement) and MLUST 0.21 (fair agreement).

Both MUST and MLUST correlate with anthropometrics to a similar degree. However, the agreement between identification of malnourished liver patients, in this select group of individuals, by MUST and MAMC is only slight, and marginally better with fair agreement with MLUST. This study illustrates that liver disease patients are difficult to screen for malnutrition, and we must be cognisant of factors that may hinder MUST such as those factors used in MLUST, a potentially useful screening tool that requires validation against outcome. 